\title{
Treating Diabetes from Cold
}

\author{
Qingxiao You', Ningjing $\mathrm{Wu}^{1}$, Zongliang Song2* \\ ${ }^{1}$ Shaanxi University of Traditional Chinese Medicine, Xianyang 712000, Shaanxi Province, China \\ 2Department of Neurology, The First People's Hospital of Changde City, Changde 415000, Hunan Province, China
}

*Corresponding author: Zongliang Song, 1023353920@qq.com

\begin{abstract}
Diabetes refers to the disease with the main symptom of "three more and less." Traditional Chinese medicine (TCM) regards Yin deficiency and dryness heat as the basic pathogenesis, and mostly adopts the method of clearing away heat, nourishing yin and generating fluid. The author thinks that the deficiency of Yuanyang in Zang Fu organs, the lack of stimulation and promotion, the metabolic disorder of body fluid, the loss of moistening of Zang Fu organs, and the onset of diabetes. Body fluid metabolism is closely related to the spleen and kidney. Deficiency of Yang Qi is the root of thirst, stagnation of body fluid and Qi is the pathogenesis, and thirst is the final result.
\end{abstract}

Keywords: Quench thirst; Treatment from cold; Warming kidney and strengthening spleen

Publication date: July 2021; Online publication: July 31, 2021

\section{Theory of treating diabetes from cold}

The theory of cold dispelling is first seen in Neijing. "The heart moves cold in the lung, the lung disappears. If the lung disappears, drink one and two." It is considered that the lung Qi is cold, the propaganda is not enough, the cold falls excessively, the water returns to the bladder, and the hair is polyuria. Kidney is the origin of congenital, which contains Yuanyang. Yuanyang is insufficient, body fluid does not melt, and the upper part does not moisten the lung. Zhong Jing in Synopsis of the Golden Chamber, "Men's thirst, urine instead of more, to drink a bucket, a bucket of urine, kidney Qi pill." Chuangshenqi pill is the first to treat diabetes by "Warming and Tonifying Kidney Yang." Later doctors mostly used heat clearing, Yin nourishing and fluid generating to treat thirst, but they did not abandon the method of warming yang. Wang Tao of Tang Dynasty said in waitai miyao, "Thirst, the primary disease, the responsibility of kidney deficiency caused by waist and kidney both deficiency and cold, cannot be steamed in the upper, Valley gas is to do the next, also for urination." It is believed that kidney yang deficiency and decline, water transpiration and gasification disorders, body fluid cannot be normal distribution, the essence of the material flow down, so urine more and taste sweet. At the same time, Sun Simiao, a physician, has many records on the application of Warming Yang drugs to treat diabetes in his book "preparation of emergency Qianjin Yaofang. Xiaoke Linbi prescription. Xiaoke No.1," such as "Xiaozhong diuretic Qiaosheng prescription" for treating diurnal polyuria. Antlers to scorch, at the end, to wine service five dagger, two days. In the treatment of Xiaoke drink more, "daily drink a stone water" application aconite, and with the season dosage. Sun Simiao not only used the combination of Warming Yang drugs to treat diabetes, but also thought that the unit warming Yang drugs had the effect of treating diabetes. For example, many warming Yang drugs in qianjinyi prescription could treat diabetes. For example, in "Volume II. Materia medica. Jade department top grade" contains quartz, sweet, warm, main thirst. In "Volume 19. Miscellaneous diseases. Diet does not disappear" Chapter 7, taiyibai pill is mainly used for Xiaogu, and its composition includes Aconitum carmichaeli. In the Ming Dynasty, Zhang Jingyue proposed that "Yang deficiency and fire deficiency lead to elimination." In the book of Jingyue, it is stated that "if Yang does not transform Qi, then water essence 
will not be distributed. It is the deficiency of true Yang, and fire deficiency leads to elimination." At the same time, Li zhongzi, a physician, proposed that Sanxiao should be based on the kidney, Bushen Shengjin should be the basis of treatment, and Wenbu Mingmen fire should be used to treat Sanxiao." The body fluid of the five Zang organs is derived from the kidney, thus Shenqi Pill is a good prescription for diabetes. It can be seen from the above that there is no obvious abnormality in the symptoms of diabetes caused by Yin deficiency and dryness heat. Therefore, we believe that diabetes can be treated by Warming Yang. ${ }^{[1][2][3][4][5]}$

Huang Yuanyu, a modern physician, thinks that kidney yang is weak, fire is not warm, spleen is weak, the Qi of liver and kidney is not rising, liver wood is trapped in water, depression leads to heat and wind, wood fire leads to gold, burns lung fluid, dry mouth and excessive drinking; Kidney water alone cold in the next, warm loss of division, bladder water gasification disorders, is polyuria. The disease of diabetes is marked by liver, which is due to kidney deficiency. From this we can see that the cause of diabetes is deficiency of kidney yang. The disease is in the spleen and kidney, and the water and body fluid are not distributed. ${ }^{[6]}$

Kidney is the innate origin, which is stored by the essence of five Zang organs. The essence of Qi is divided into Yin and Yang, and the Yang is divided into Yin and Yang. If the kidney yang is lost, it will be lost in transpiration and gasification of water, and the body fluid will not reach the lung, and the lung will not propagate the body fluid around the body, and the bones will not moisten, and dry mouth and excessive drinking can be seen in Shangjiao; Shangjiao is like fog. It spreads the body fluid to moisten Yangming Fu organs. Yangming has many channels of Qi and blood. It likes moistening and evils dryness. If the lung fluid does not moisten Yangming, and the stomach Yang is hot, it will eliminate the valley and relieve hunger "Two Yang knot is called the elimination," The deficiency of kidney yang, the weakness of bladder gasification, the loss of water and liquid solid intake, the direct infusion of water and grain essence into the bladder, so we can see polyuria and turbid urine. ${ }^{[7]}$

Spleen and stomach are the hub of qi movement, the source of Qi and blood, the foundation of postnatal, and the mutual promotion of congenital and postnatal. The metabolism of body fluid is closely related to lung, spleen, kidney and triple energizer "Drinking into the stomach, infusing into the spleen, dispersing the essence of the spleen, and returning to the lung; The five meridians are parallel." The metabolic transport process of body fluid in vivo was described in detail. The channel of Sanjiao's water and liquid operation is stated in Suwen Linglan's Secret treatise." The triple energizer is the official who decides to violate the law." Kidney yang deficiency, fire not warm soil, spleen deficiency not transport, body fluid stopped, four side loss of nourishment, in the upper Jiao lung body fluid xuanjiang disorder, hair dry mouth, drink more; Kidney yang deficiency, kidney water only cold in the lower, spleen soil has declined, kidney water insulting soil, aggravating spleen cold, water transport disorders, spleen not for the stomach line its body fluid, in the middle Jiao performance for food but not hunger, drink a few not quench thirst; Qi deficiency subsides, the Qi of liver and kidney does not rise, forming the syndrome of soil blocking and wood stagnation, liver blocking, abnormal catharsis, Sanjiao blocking, body fluid failing, viscera not moistening. To sum up, the basic disease of diabetes is kidney yang deficiency, and the pathogenesis is Yang deficiency, body fluid is not distributed, and the viscera is not moistened. Among them, Jinting is drinking, blocking Qi, depression and heat, internal injury to body fluid, aggravating the deficiency of viscera and body fluid, and all these factors contribute to diabetes. The treatment should be based on the spleen and kidney, warming the kidney and benefiting the spleen, removing dampness and eliminating Yin, and smoothing Qi.

\section{Disclosure statement}

The author declares no conflict of interest. 


\section{References}

[1] Yu S, 2006, Huangdi Neijing. Chaohua Press, Beijing.

[2] Wang T, 1955, Wai Tai Mi Yao. Beijing: People's Health Publishing House, 30317.

[3] Sun S, 2010, Qian Jin Yi Fang. Taiyuan, China. Shanxi Science and Technology Publishing House, 30602 .

[4] Tian Z, 2003, Discussion on Zhang Jingyue's Academic Thought of Diabetes. Henan Traditional Chinese Medicine, 23(9): 13.

[5] Li Z, 1985, Zheng Zhi Hui Bu. Shanghai Health Press, Shanghai, 318.

[6] Li J, Guo K, Zhou S, et al., 2017, Huang Yuanyu's Theory of Treating Diabetes from Liver, Spleen and Kidney. Jiangsu Traditional Chinese Medicine, 5: 13-14.

[7] Liu D, 1991, Notes on Treatise on Febrile Diseases. China People's Health Publishing House, Beijing. 147, 135, 59, 99, 195. 